Archive for

Organic Chemistry

Arkivoc 2019, part ii, 163-175

\title{
Synthesis and biological evaluation of new indole and pyrrole carboxamides based on amino acids
}

\author{
Martina Palomba, Sara Pompei, Luca Roscini, and Luana Bagnoli* \\ Department of Pharmaceutical Sciences \\ University of Perugia, Via del Liceo 1, 06123 Perugia, Italy \\ Email: luana.bagnoli@unipg.it
}

\section{Dedicated to Professor Lorenzo Testaferri in the occasion of his $75^{\text {th }}$ birthday}

Received 10-28-2019

Accepted 12-19-2019

Published on line $01-05-2020$

\section{Abstract}

A new series of indole carboxamides have been synthesized through coupling reactions. Several substitutions on the aromatic ring and on the amino acids are well tolerated, and the corresponding indole carboxamides have been obtained with good yields. The same procedure can be also extended to the pyrrole nucleus. Four of these compounds have been screened for their antimicrobial activity against ten different yeast strains and two of them showed an antifungal activity. Further explorations are required to clarify a potential applicability in biological fields.

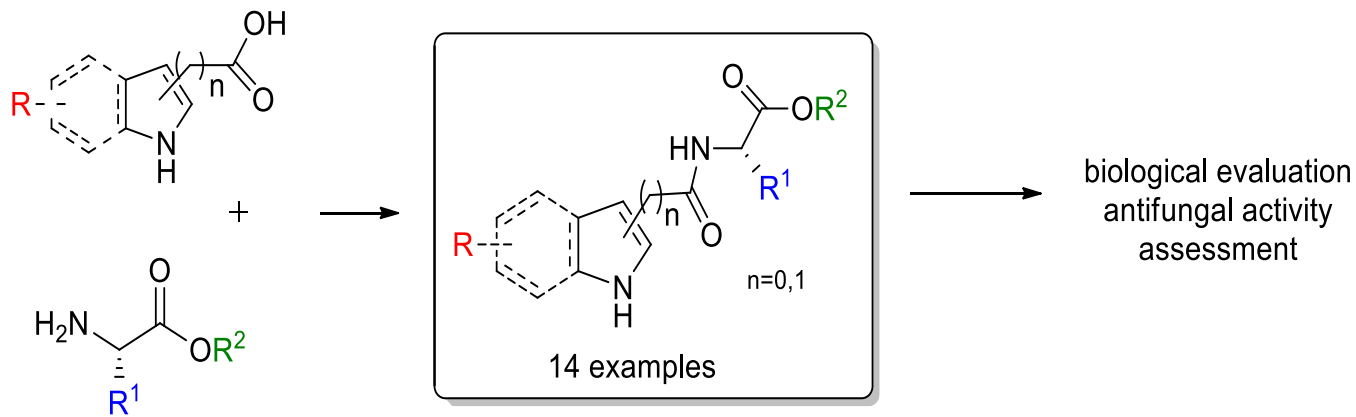

Keywords: Amino acid, carboxamide, indole, pyrrole, amide bond. 


\section{Introduction}

Amide bond plays a key role in the development and the composition of many biological systems, be they natural or synthetic molecules. ${ }^{1-3}$ For example, it is present in some proteins that play a crucial role in enzyme catalysis, in blood transport/storage processes (haemoglobin), in immune protection (antibodies) and in the mechanical support (collagen). Amides are also widely employed in the preparation of fine chemicals, cosmetics, food additives and pharmaceutical drugs. Detailed analyses reported by the Comprehensive Medicinal Chemistry database found that more than $25 \%$ of common drugs bear amide functionality. ${ }^{4-6}$ For example Penicillin, a bacterial antibiotic, Taxol, an anticancer agent, Lisinopril, one of the inhibitors of Angiotensin, Valsartan, an antihypertensive and Atorvastatin (Lipitor) that is used to treat high cholesterol. Therefore, it is not surprising that in 2007 the American Chemical Society Green Chemistry Institute Pharmaceutical Roundtable voted "amide formation" as the priority area of research for the pharmaceutical industry. In fact, the $65 \%$ of all preliminary screening reactions in industrial medicinal chemistry laboratories employ the amide linkages. ${ }^{7-9}$ This is due to the favourable properties of carboxamides being neutral, stable and able to be acceptors or donors of hydrogen bonds. In addition, their high bonding polarity as well as their conformational diversity make them one of the most popular functional groups of organic chemistry.

The amide function linked to an indole nucleus is particularly important in the pharmaceutical field. Indole is a privileged structure in various research areas such as: pharmaceuticals, agrochemicals and organic functional material science. The broad spectrum of applications of the indole justifies it being addressed as the "Lord of the Ring" ${ }^{10}$ In particular, indole-2-carboxamide scaffolds are known to have significant biological activity such as antitumor, ${ }^{11-13}$ anti-infiammatory, ${ }^{14-16}$ antitubercular, ${ }^{17-19}$ antibacterial ${ }^{20-22}$ and antifungal ${ }^{23-26}$ (Figure 1). A possible anti-tumor activity is described through growth factor inhibition for kinase tyrosine protein receptors (PTKs), which are found over-expressed in cancer cells. ${ }^{12}$ Indolylarylsulfone carboxamides carrying a heterocyclic tail are reported as potential anti-HIV-1/AIDS agents, ${ }^{27}$ and other $1 \mathrm{H}$-indole-2-carboxamide derivatives were discovered as novel inhibitors of the androgen receptor binding function 3 ( AR-BF3) showed an antiproliferative activity against wild type and drug resistant prostate cancer cells ${ }^{28}$ (Figure 1). Several studies of structure-active relationships (SAR) on indole based carboxamides are reported in the literature relate to activity as inhibitors of the Hepatitic C virus (HCV) NS5B polymerase ${ }^{29}$ or inhibitory mode against human liver glycogen phosphorylase (HLGPa). ${ }^{30}$ Furthermore, some papers report for indole carboxamides ${ }^{31}$ and pyrrole carboxamides ${ }^{32,33}$ an inhibitory capacity of MAO-A, which is useful for the treatment of depression and anxiety states, and MAO-B, exploited in the treatment of Parkinson's disease. Based on the literature data concerning the promising potential of the indole carboxamides, our idea was to combine two biologically relevant scaffolds such as indole and various natural amino acids. Only sporadic examples are reported in the literature for the indole carboxamides based on amino acids. These investigations are related to their agonist activity at the level of NMDA receptor subtype (GluN1/2A-D), expressed at the level of the central nervous system. ${ }^{34} \mathrm{~A}$ series of dipeptide indole carboxamides containing indole-2-carbonyl unit display potent inhibitory activity against the severe acute respiratory syndrome promoted by Coranovirus (SARS-COV $3 \mathrm{Cl}$ ). ${ }^{35}$ These compounds can be particularly attractive under pharmaceutical profile because are peptidomimetic substrates. 
<smiles>O=C(Nc1cccc(-c2ccccc2)c1)c1cc2ccccc2[nH]1</smiles>

Anticancer<smiles>[R][R]1cccc(-n2c(C(=O)N([R])[R])cc3cc(S(C)(=O)=O)ccc32)c1</smiles>

Anti-inflammatory

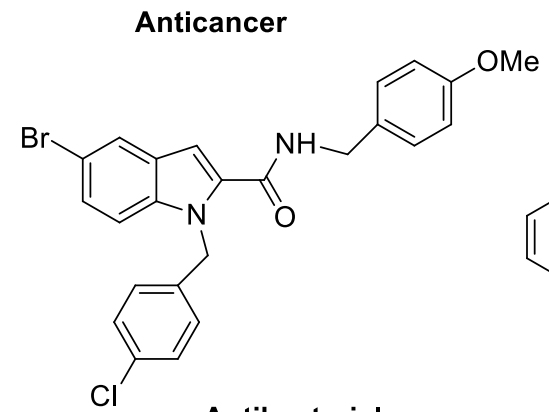

Antibacterial<smiles>Cc1ccc(C(NC(=O)c2cc3ccc(F)cc3[nH]2)C(C)C)o1</smiles>

Antiandrogen<smiles>CC(C)OC(=O)[C@H](Cn1ccnc1)NC(=O)c1cc2ccccc2n1C</smiles><smiles>Cc1cc(C)c2cc(C(=O)NC3CCCCC3)[nH]c2c1</smiles>

Antitubercular<smiles>Cc1cc(C)cc(S(=O)(=O)c2c(C(=O)N[C@H](C)c3ccncc3)[nH]c3ccc(Cl)cc23)c1</smiles>

Antifungal<smiles>CN(Cc1ccccc1)C(=O)c1cc2ccccc2[nH]1</smiles>

MAO-A inhibitor

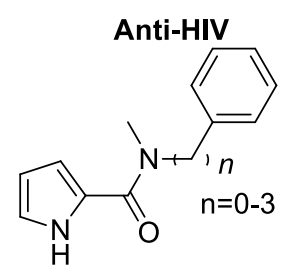

MAO-B inhibitor<smiles>Cc1cc2c(-c3ccc[nH]c3=O)c(C(=O)NS(=O)(=O)C3CC3)n(Cc3cc(F)ccc3F)c2cc1F</smiles>

Inhibitor HCV NS5B<smiles>N[C@@H](CNC(=O)c1cc2ccccc2[nH]1)C(=O)O</smiles>

GluN2C Agonist at GluN1

Figure 1. Examples of reported bioactive indole-2-carboxamides.

Herein we report the synthesis of a new series of indole or pyrrole carboxamides based on amino acids. Some of these derivatives have been screened for possible antimicrobial and antifungal activities against ten different yeast strains. Eight of them belongs to the four species of the genus Candida, an opportunistic fungal genus presents in both medical and environmental fields and responsible for different types of infections, with the higher isolation frequency: Candida albicans, Candida glabrata, Candida parapsilosis and Candida tropicalis. The other species considered in our study are Meyerozyma guilliermondii, a member of the same genus isolated in fewer cases, but still capable of causing major infections; and Saccharomyces cerevisiae, which represents the study model for microbial eukaryotes, chosen as an outgroup. Due to limited number of studies of the effect of these compounds against yeasts, this screening represents a quite new field of application of these derivatives. 


\section{Results and Discussion}

Various methods for amidic formation have attracted the attention of organic chemists and a variety of synthetic strategies have been developed and optimized. ${ }^{36-41}$ The formation of the amidic bond involved amino acids often occurs with difficulty as it can lead to low yields, racemizations of chiral centres, degradation of products and complications during the purification. The synthesis of indole and pyrrole 2- or 3carboxamides were carried out via a coupling reaction by combining indolic or pyrrolic nucleus with various natural amino acids (Schema 1). The reaction initially proceeds with the addition of $N, N$ dicyclohexylcarbodimmide (DCC) to indole or pyrrole carboxylic acids in tetrahydrofuran (THF) giving to the intermediate O-acylurea. ${ }^{37}$ After 10 minutes, the addition of $\mathrm{N}$-hydroxybenzotriazole (HOBt) leads to the formation of the corresponding activated ester. Meanwhile, a suspension of the amino acids and trimethylamine in dry tetrahydrofuran was prepared and added by dropping. Studies were performed by Konig and Geiger demonstrated that the introduction of $\mathrm{HOBt}$ as an additive to DCC reduces the racemization. ${ }^{42-43}$

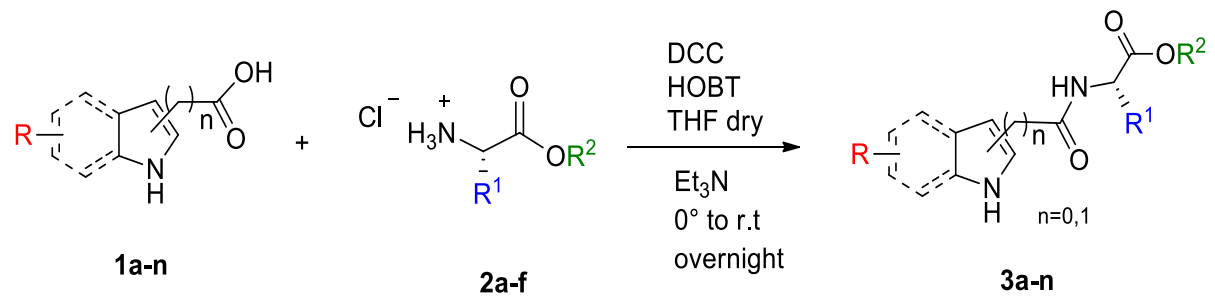

Scheme 1. Coupling reaction.

Initially, the study was conducted using indole-2-carboxylic acid, 1a and several amino acids, 2a-f as starting substrates. (Table 1)

Table 1. Variation of the amino acid chains

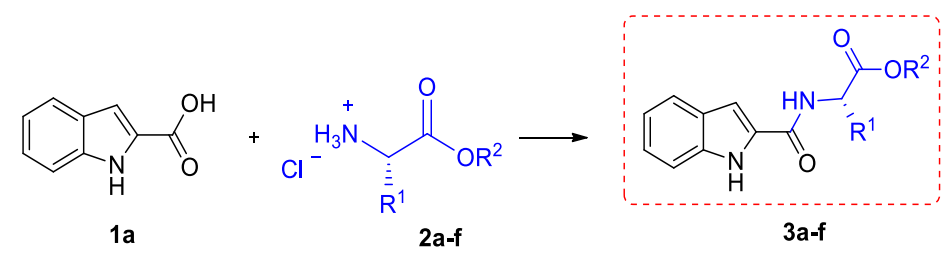

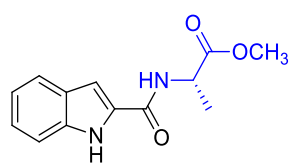

$3 a, 70 \%$

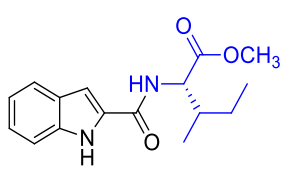

$3 d, 49 \%$

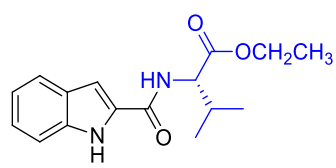

3b, $65 \%$

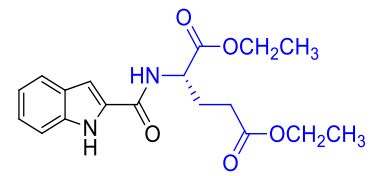

3e, $57 \%$

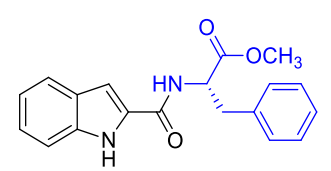

3c, $50 \%$

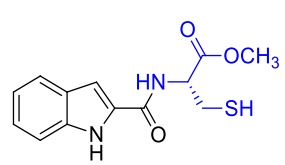

3f, $61 \%$ 
The products 3a-f were obtained with moderate to good yields. Using L-alanine methyl ester hydrochloride and L-valine ethyl ester hydrochloride, the final products $\mathbf{3 a}$ and $\mathbf{3 b}$ were obtained with a yield of $70 \%$ and $65 \%$ respectively. Subsequently starting from amino acids, bearing in the side chain sterically bulky groups, such as L-phenylalanine methyl ester hydrochloride $\mathbf{2 c}$ and the L-isoleucine methyl ester hydrochloride $\mathbf{2 d}$, the respective products $\mathbf{3 c}$ and $\mathbf{3 d}$ were obtained in moderate to good yields. In order to evaluate the generality and robustness of the method, amino acids, which have in the side chain other functional groups such as ester and thiol, were employed. Even in these cases, starting from L-glutamic acid diethyl ester hydrochloride $\mathbf{2 e}$ and L-cysteine methyl ester hydrochloride $\mathbf{2} \mathbf{f}$ the reactions proceed towards the formation of the carboxamides $3 \mathbf{e}$ and $\mathbf{3 f}$ in $57 \%$ and $61 \%$ yields respectively.

Next, the effects of the substituents and the position of the carbonyl unit in the indole nucleus and also the insertion of pyrrole were evaluated (Table 2).

Table 2. Structural changes to the indole and pyrrole nuclei

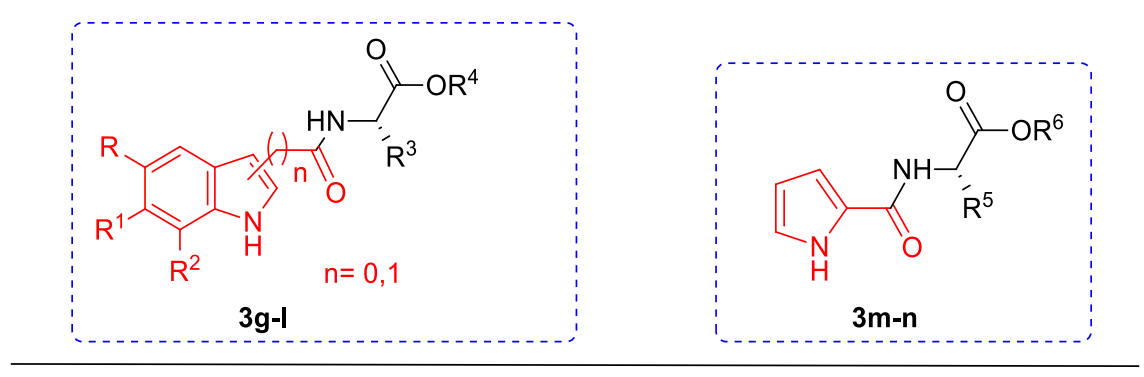

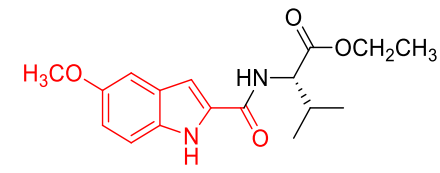

$3 g, 53 \%$

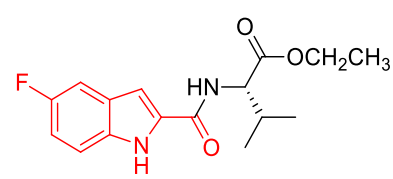

3i, $47 \%$

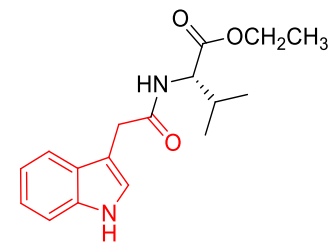

3k, $44 \%$

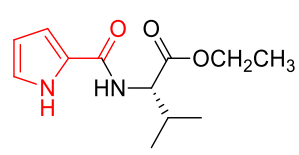

3m, 65\%

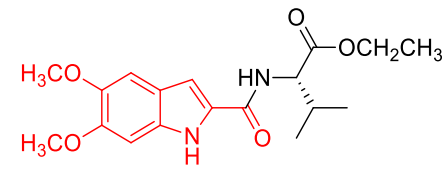

3h, $64 \%$

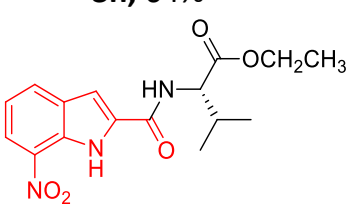

3j, 79\%

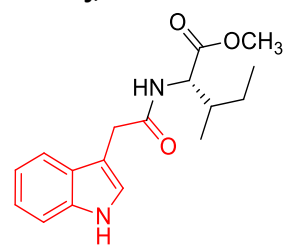

3I, $72 \%$

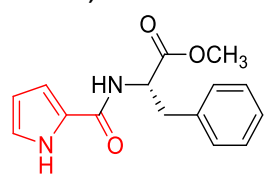

$3 n, 58 \%$

Various substituents in the 5, 6 or 7 positions of the indoles have been employed. Both electron-donating groups (EDG) such as methoxy $(\mathbf{3 g}, \mathbf{3 h})$, and electron-withdrawing group (EWG) such as fluoride (3i) were tolerated. Better result was obtained using 5-nitro indol-2-carboxilic acid as a starting material, which led to 
the product $\mathbf{3} \mathbf{j}$ with a yield of $79 \%$. In addition, we evaluated the replacement of the carboxylic group from the 2 to the 3 position on the indole core. Starting from $1 \mathrm{H}$-indole-3-yl acetic acid, the formation of products $\mathbf{3 k}$ and $\mathbf{3} \mathbf{l}$ were obtained with more or less variable yields. Using L-valine ethyl ester hydrochloride the product $\mathbf{3 k}$ was obtained in $44 \%$ yield, while using the L-isoleucine methyl ester hydrochloride the product 3 I was isolated in $72 \%$ yield. Next, we extended the method to the pyrrole nucleus. Two sets of amino acids were used, the Lvaline ethyl ester hydrochloride and the L-phenylalanine methyl ester hydrochloride, obtaining the pyrrole carboxamides derivatives $\mathbf{3 m}, \mathbf{3 n}$ in $65 \%$ and $58 \%$ yields, respectively. Some of these derivatives (3b, $\mathbf{3 g}$ and $3 \mathrm{~m}$ ) have been used with success in the Michael Initiated Ring Closure Reaction (MIRC) for the synthesis of enantiopure pyrazino fused-indoles and pyrroles without racemization. ${ }^{44}$

Given the shortage of evaluation of possible biological significance of these types of molecules in literature, the growth inhibition abilities of some of these indole and pyrrole carboxamides (3g, $\mathbf{3 h}, \mathbf{3 j}, \mathbf{3 m}$ ) were evaluated by MIC (Minimum Inhibitory Concentration) assay against ten fungal strains belonging to different species. Only compounds $\mathbf{3} \mathbf{j}$ and $\mathbf{3 m}$ showed a clear antimicrobial activity. MIC values were always above 62.5 $\mu \mathrm{g} / \mathrm{mL}$, for all strains used in this valuation, a concentration that is higher than those normally shown by antifungal drugs already in use in clinical therapy. Compounds $\mathbf{3 g}$ and $\mathbf{3 h}$ showed a lower, although present, antimicrobial activity.

\section{Conclusions}

In conclusion, we developed the synthesis of new differently functionalized carboxamides obtained through a coupling reaction between 2- or 3-indole carboxylic acid or pyrrole 2-carboxylic acid with various amino acids. Several substitutions both on the aromatic ring and on the amino acid are well tolerated, and the corresponding carboxamides were obtained with satisfactory yields.

Some of these compounds have been screened for their antifungal activity but these preliminary biological evaluations highlighted that compounds $\mathbf{3} \mathbf{j}$ and $\mathbf{3} \mathbf{m}$ have an inhibitory capacity against microbial growth at concentrations higher than $62.5 \mu \mathrm{g} / \mathrm{mL}$, a concentration higher than the ones normally used for the antimycotics. Lower, although not absent, is the inhibitory activity shown by compounds $\mathbf{3 g}$ and $\mathbf{3 h}$, in the same concentration range. Given the presence within the same structure of two interesting nuclei, further work is in progress to explore other potential biological applications of these carboxamides based on amino acids.

\section{Experimental Section}

Chemistry. Commercial reagents and solvents were purchased from Sigma Aldrich, Alfa Aesar or VWR International and used without further purification. Thin layer chromatography (TLC) was performed on silica gel $60 \mathrm{~F}_{254}$ (Merck) on aluminum sheets. Reaction products were purified by column chromatography performed on Merck silica gel 60 (70-230 mesh). ${ }^{1} \mathrm{H}$ and ${ }^{13} \mathrm{C}-\mathrm{NMR}$ spectra were recorded at 400 and 100.62 $\mathrm{MHz}$, respectively, on a Bruker Avance-DRX 400 instrument. Chemical shifts $(\delta)$ are reported in parts per million related to residual solvent signals $\left(\mathrm{CHCl}_{3}, 7.26 \mathrm{ppm}\right.$, DMSO $2.49 \mathrm{ppm}$ for ${ }^{1} \mathrm{H} \mathrm{NMR,} \mathrm{CDCl}_{3} 77.0 \mathrm{ppm}$, DMSO 39.7 ppm for ${ }^{13} \mathrm{C}$ NMR). Coupling constants are given in Hertz ( $\left.\mathrm{Hz}\right)$. The following abbreviations are used to indicate the multiplicity: $s$, singlet; $d$, doublet; $t$, triplet; $m$, multiplet; quint, quintet; sex, sextet; brs, broad 
signal. FT-IR spectra were recorded with a Jasco model 410 spectrometer equipped with a diffuse reflectance accessory and with Shimadzu QATR-S model. High resolution mass spectra (HRMS) were recorded on an Agilent 6540-UHD Accurate Mass Q-TOF LC/MS instrument or APEX IV 7T FTICR. GC analyses and MS spectra were carried out with an HP 6890 gas chromatograph (25 m dimethyl silicone capillary column) equipped with an HP 5973 Mass Selective. Optical rotations were measured with AP-100 automatic polarimeter Atago.

Synthesis of indole and pyrrole carboxamides, 3a-n. To a suspension of indole or pyrrole carboxylic acids $1 a-h$ $(1.0 \mathrm{mmol})$ in dry tetrahydrofuran $(8 \mathrm{~mL}) \mathrm{N}, N^{\prime}$-dicyclohexylcarbodiimmide $(1.5 \mathrm{mmol})$ was added portionwise, maintaining the temperature below $0^{\circ} \mathrm{C}$. After 20 minutes, 1-hydroxybenzotriazole $(1.5 \mathrm{mmol})$ in dry tetrahydrofuran $(4 \mathrm{~mL})$ was added, and the reaction was allowed to stir for additional 20 minutes. Finally, a suspension of desired amino ester hydrochloride $(1.5 \mathrm{mmol})$ and triethylammine in dry tetrahydrofuran was prepared and added dropwise. The reaction mixtures were allowed to warm to room temperature. The progress of the reactions was monitored by TLC. The reaction mixture was filtrated in vacuo and the filtrate was diluted and extracted with EtOAc $(3 \times 50 \mathrm{ml})$ and washed with $5 \% \mathrm{NaHCO}_{3}, 10 \% \mathrm{HCl}$, water, and finally with brine. The organic layers were dried with anhydrous sodium sulfate and evaporated in vacuo. The products were purified using column chromatography on silica gel (EtOAc/PE 80:20 as eluent) afford the indole carboxamides 3a-I and pyrrole carboxamides $\mathbf{3 m}-\mathbf{n}$. The spectroscopic data of compounds 3a-n are reported below. For known compounds $\mathbf{3 a - b}, \mathbf{g}, \mathbf{m}-\mathbf{n}$ only ${ }^{1} \mathrm{H}$ and ${ }^{13} \mathrm{C}$ NMR are reported. ${ }^{44,45}$

Methyl (2S)-2-[(1H-indol-2-ylcarbonyl)amino]propanoate, 3a: white solid; yield 70\%; m.p. $128-132^{\circ} \mathrm{C} .{ }^{1} \mathrm{H}$ NMR $\left(400 \mathrm{MHz}, \mathrm{CDCl}_{3}, 25^{\circ} \mathrm{C}, \mathrm{TMS}\right): \delta=9.30$ (brs, $\left.1 \mathrm{H}\right), 7.68(\mathrm{~d}, 1 \mathrm{H}, J 8.0 \mathrm{~Hz}), 7.45(\mathrm{~d}, 1 \mathrm{H}, J 8.3 \mathrm{~Hz}), 7.31(\mathrm{t}, 1 \mathrm{H}, J 8.0$ $\mathrm{Hz}$ ), $7.16(\mathrm{t}, 1 \mathrm{H}, J 8.0 \mathrm{~Hz}$ ), $6.96(\mathrm{~d}, 1 \mathrm{H}, J 1.2 \mathrm{~Hz}), 6.80$ (brd, 1H, J $6.4 \mathrm{~Hz}), 4.85$ (quint, $1 \mathrm{H}, J 7.2 \mathrm{~Hz}), 3.82(\mathrm{~s}, 3 \mathrm{H})$, $1.57(\mathrm{~d}, 3 \mathrm{H}, \mathrm{J} 7.1 \mathrm{~Hz}) ;{ }^{13} \mathrm{C} \mathrm{NMR}\left(100 \mathrm{MHz} \mathrm{CDCl}_{3}\right): \delta=174.0,162.1,136.8,130.0,127.4,124.8,122.2,120.6$, 112.6, 103.5, 53.4, 48.6, 18.3.

Ethyl (2S)-2-[(1H-indol-2-carbonyl)amino]-3-methylbutanoate, 3b: white solid; yield 65\%; m.p. $109-114^{\circ} \mathrm{C} .{ }^{1} \mathrm{H}$ NMR (400 MHz, $\left.\mathrm{CDCl}_{3}, 25^{\circ} \mathrm{C}, \mathrm{TMS}\right): \delta=10.25$ (brs, $\left.1 \mathrm{H}\right), 7.69(\mathrm{~d}, 1 \mathrm{H}, J 8.0 \mathrm{~Hz}), 7.50$ (d, $\left.1 \mathrm{H}, J 8.0 \mathrm{~Hz}\right), 7.32(\mathrm{t}, 1 \mathrm{H}, J$ $8.0 \mathrm{~Hz}), 7.16(\mathrm{t}, 1 \mathrm{H}, J 8.0 \mathrm{~Hz}), 7.05(\mathrm{~d}, 1 \mathrm{H}, J 1.2 \mathrm{~Hz}), 7.0(\mathrm{~d}, 1 \mathrm{H}, J 8.7 \mathrm{~Hz}), 4.87(\mathrm{dd}, 1 \mathrm{H}, J 5.2,8.7 \mathrm{~Hz}), 4.36-4.25$ $(\mathrm{m}, 2 \mathrm{H}), 2.42-2.31(\mathrm{~m}, 1 \mathrm{H}), 1.35(\mathrm{t}, 3 \mathrm{H}, J 7.0 \mathrm{~Hz}), 1.08(\mathrm{t}, 6 \mathrm{H}, J 7.2 \mathrm{~Hz}) ;{ }^{13} \mathrm{C} \mathrm{NMR}\left(100 \mathrm{MHz}, \mathrm{CDCl}_{3}\right): \delta=172.1$, 161.7, 136.7, 130.1, 127.4, 124.4, 121.9, 120.5, 112.1, 102.8, 61.5, 57.3, 31.6, 19.0, 18.0, 14.2.

Methyl (2S)-2-[(1H-indol-2yl-carbonyl)amino]-3-phenylpropanoate, 3c: white solid; yield 50\%; m.p. 123$\left.126^{\circ} \mathrm{C} ; \alpha\right]_{D}^{21}=+32.5$ (c 1.2, $\mathrm{CHCl}_{3}$ ). ${ }^{1} \mathrm{H} \mathrm{NMR}\left(400 \mathrm{MHz} \mathrm{CDCl}_{3}, 25^{\circ} \mathrm{C}, \mathrm{TMS}\right): \delta=10.47$ (brs, $\left.1 \mathrm{H}\right), 7.67(\mathrm{~d}, 1 \mathrm{H}, J 8.0$ $\mathrm{Hz}), 7.51(\mathrm{~d}, 1 \mathrm{H}, J 8.0 \mathrm{~Hz}), 7.40-7.23(\mathrm{~m}, 6 \mathrm{H}), 7.20-7.14(\mathrm{~m}, 2 \mathrm{H}), 6.96(\mathrm{~d}, 1 \mathrm{H}, J 1.9 \mathrm{~Hz}), 5.30-5.23(\mathrm{~m}, 1 \mathrm{H}), 3.83(\mathrm{~s}$, $3 \mathrm{H}), 3.40(\mathrm{dd}, 1 \mathrm{H}, J 5.8,13.8 \mathrm{~Hz}), 3.33(\mathrm{dd}, 1 \mathrm{H}, J 6.2,13.8 \mathrm{~Hz}) ;{ }^{13} \mathrm{C} \mathrm{NMR}\left(100 \mathrm{MHz}, \mathrm{CDCl}_{3}\right): \delta=172.3,161.7$, $136.9,135.8,130.0,129.3$ (2C), 128.7(2C), 127.5, 127.3, 124.5, 121.9, 120.5, 112.3, 103.2, 52.5, 51.8, 38.1; MS (70 ev, El): m/z (\%) 199 (100) [M+], 291 (22), 290 (90), 144 (25), 115 (20), 91 (95), 89 (26); FT-IR (KBr) U max 3356, $3299,1728,1635,1539 \mathrm{~cm}^{-1}$.

Methyl (2S,3S)-2-[(1H-indol-2-ylcarbonyl)amino]-3-methylpentanoate, 3d: white solid; yield 49\%; m.p. 135$139^{\circ} \mathrm{C} ;[\alpha]_{D}^{21}=-26.5$ (c 1.0, $\left.\mathrm{CHCl}_{3}\right) .{ }^{1} \mathrm{H}$ NMR $\left(400 \mathrm{MHz}, \mathrm{CDCl}_{3}, 25^{\circ} \mathrm{C}, \mathrm{TMS}\right): \delta=9.37$ (brs, $\left.1 \mathrm{H}\right), 7.69(\mathrm{~d}, 1 \mathrm{H}, J 8.1$ $\mathrm{Hz}), 7.46(\mathrm{~d}, 1 \mathrm{H}, J 8.3 \mathrm{~Hz}), 7.32(\mathrm{t}, 1 \mathrm{H}, J 8.1 \mathrm{~Hz}), 7.17(\mathrm{t}, 1 \mathrm{H}, J 8.0 \mathrm{~Hz}), 6.98(\mathrm{~d}, 1 \mathrm{H}, J 1.4 \mathrm{~Hz}), 6.73(\mathrm{brd}, 1 \mathrm{H}, J 8.4$ $\mathrm{Hz}), 4.86(\mathrm{dd}, 1 \mathrm{H}, J 5.1,8.6 \mathrm{~Hz}), 3.81(\mathrm{~s}, 3 \mathrm{H}), 2.09-2.0(\mathrm{~m}, 1 \mathrm{H}), 1.63-1.52(\mathrm{~m}, 1 \mathrm{H}), 1.35-1.25(\mathrm{~m}, 1 \mathrm{H}), 1.04-0.97$ $(\mathrm{m}, 6 \mathrm{H}) ;{ }^{13} \mathrm{C}$ NMR $\left(100 \mathrm{MHz}, \mathrm{CDCl}_{3}\right): \delta=172.6,161.1,136.2,129.9,127.6,124.8,122.0,120.7,111.9,102.6$, 56.5, 52.3, 38.7, 25.2, 15.7, 11.5; FT-IR (KBr) $u_{\max }$ 3298, 2927, 1732, 1637, $1550 \mathrm{~cm}^{-1}$; HRMS (ESI-TOF) $m / z$ : $[\mathrm{M}+\mathrm{H}]^{+}$calcd for $\mathrm{C}_{16} \mathrm{H}_{21} \mathrm{~N}_{2} \mathrm{O}_{3}$ 289.1552; found: 289.1553 .

Diethyl (2S)-2-[(1H-indol-2yl-carbonyl)amino]pentanedioate, 3e: colorless oil; yield $57 \%$; $[\alpha]^{21}=+20.0$ (c 1.0, $\left.\mathrm{CHCl}_{3}\right) .{ }^{1} \mathrm{H} \mathrm{NMR}\left(400 \mathrm{MHz}, \mathrm{CDCl}_{3}, 25^{\circ} \mathrm{C}, \mathrm{TMS}\right): \delta=9.80$ (brs, $\left.1 \mathrm{H}\right), 7.68(\mathrm{~d}, 1 \mathrm{H}, J 8.0 \mathrm{~Hz}), 7.47(\mathrm{~d}, 1 \mathrm{H}, J 8.0 \mathrm{~Hz})$, 7.31-7.27 (m, 2H), $7.15(\mathrm{t}, 1 \mathrm{H}, J 8.0 \mathrm{~Hz}), 7.01(\mathrm{~d}, 1 \mathrm{H}, J 1.0 \mathrm{~Hz}), 4.89-4.82(\mathrm{~m}, 1 \mathrm{H}), 4.27(\mathrm{q}, 2 \mathrm{H}, J$ 7.1 Hz), 4.18-4- 
$09(\mathrm{~m}, 2 \mathrm{H}), 2.62-2.44(\mathrm{~m}, 2 \mathrm{H}), 2.41-2.31(\mathrm{~m}, 1 \mathrm{H}), 2.26-2.15(\mathrm{~m}, 1 \mathrm{H}), 1.32(\mathrm{t}, 3 \mathrm{H}, J 7.1 \mathrm{~Hz}), 1.23(\mathrm{t}, 3 \mathrm{H}, J 7.1 \mathrm{~Hz})$; ${ }^{3} \mathrm{C}$ NMR $\left(100 \mathrm{MHz}_{\mathrm{CDCl}}\right): \delta=173.5,172.0,161.9,136.9,130.0,127.5,124.4,122.0,120.3,112.3,103.3,61.8$, 60.9, 52.3, 30.5, 27.0, 14.1, 14.0; FT-IR (KBr) $u_{\max }$ 3350, 3270, 1734, 1646, $1530 \mathrm{~cm}^{-1}$. HRMS (ESI-TOF) $\mathrm{m} / \mathrm{z}$ : $[\mathrm{M}+\mathrm{H}]^{+}$calcd for $\mathrm{C}_{18} \mathrm{H}_{23} \mathrm{~N}_{2} \mathrm{O}_{5} 347.1607$; found: 347.1618 .

Ethyl (2R)-2-[(1H-indol-2ylcarbonyl)amino]-3-mercaptopropanoate, 3f: white solid; yield 61\%; m.p. 118$\left.121^{\circ} \mathrm{C} ; \alpha\right]_{\mathrm{D}}^{21}=-35.0$ (c 1.0, MeOH). ${ }^{1} \mathrm{H}$ NMR (400 MHz, DMSO $\left.d_{6}, 25^{\circ} \mathrm{C}\right) ; \delta=11.61$ (brs, $\left.1 \mathrm{H},\right), 8.91(\mathrm{~d}, 1 \mathrm{H}, J 7.9$ $\mathrm{Hz}), 7.63(\mathrm{~d}, 1 \mathrm{H}, J 7.9 \mathrm{~Hz}), 7.43(\mathrm{~d}, 1 \mathrm{H}, J 8.3 \mathrm{~Hz}), 7.23-7.16(\mathrm{~m}, 2 \mathrm{H}), 7.10-7.0(\mathrm{~m}, 1 \mathrm{H}), 4.87-4.78(\mathrm{~m}, 1 \mathrm{H}), 3.67(\mathrm{~s}$, $3 \mathrm{H}), 3.37-3.30(\mathrm{~m}, 2 \mathrm{H}), 3.19(\mathrm{dd}, 1 \mathrm{H}, J 9.8,13.6 \mathrm{~Hz}) ;{ }^{3} \mathrm{C} \mathrm{NMR}\left(100 \mathrm{MHz}, \mathrm{CDCl}_{3}\right): \delta=170.7,161.4,136.4,129.7$, 127.5, 124.9, 122.2, 120.8, 111.8, 103.7, 52.9, 52.3, 41.2; FT-IR (KBr) U $\max 3338,3256,1734,1622,1540 \mathrm{~cm}^{-1}$. HRMS (APCl-TOF) $\mathrm{m} / \mathrm{z}$ : [M+H] ${ }^{+}$calcd for $\mathrm{C}_{13} \mathrm{H}_{16} \mathrm{~N}_{2} \mathrm{O}_{3} \mathrm{~S} 279.0803$; found: 279.0799 .

Ethyl (2S)-2-\{[(5-methoxy-1H-indol-2yl)carbonyl]amino\}-3-methylbutanoate, 3g: ${ }^{44}$ white solid; yield 53\%; m.p. $105-110^{\circ} \mathrm{C} .{ }^{1} \mathrm{H}$ NMR $\left(400 \mathrm{MHz}, \mathrm{CDCl}_{3}, 25^{\circ} \mathrm{C}, \mathrm{TMS}\right): \delta=9.98$ (brs, $\left.1 \mathrm{H}\right), 7.38(\mathrm{~d}, 1 \mathrm{H}, J 8.9 \mathrm{~Hz}), 7.07(\mathrm{~d}, 1 \mathrm{H}, J$ $2.1 \mathrm{~Hz}$ ), 6.98 (dd, 1H, J 2.1, 8.9 Hz), 6.94 (d, 1H, J 1.4 Hz), 7.90-6.87 (brs, 1H), 4.83 (dd, 1H, J 5.1, 8.7 Hz), 4.36$4.21(\mathrm{~m}, 2 \mathrm{H}), 3.87(\mathrm{~s}, 3 \mathrm{H}), 2.40-2.29(\mathrm{~m}, 1 \mathrm{H}), 1.34(\mathrm{t}, 3 \mathrm{H}, J 7.1 \mathrm{~Hz}), 1.06(\mathrm{t}, 6 \mathrm{H}, J 7.1 \mathrm{~Hz}) ;{ }^{13} \mathrm{C} \mathrm{NMR}(100 \mathrm{MHz}$, $\left.\mathrm{CDCl}_{3}\right): \delta=172.1,161.6,154.5,132.0,130.5,127.8,115.9,113.0,102.4,102.1,61.5,57.3,55.6,31.7,19.0$, 18.0, 14.2 .

Ethyl (2S)-2-\{[(5,6-dimethoxy-1H-indol-2yl)carbonyl]amino\}-3-methylbutanoate, 3h: white solid; yield 64\%; m.p. $165-168^{\circ} \mathrm{C} ;[\alpha]^{20}{ }_{D}=-20.0$ (c 1.0, $\mathrm{CHCl}_{3}$ ). ${ }^{1} \mathrm{H}$ NMR $\left(400 \mathrm{MHz}, \mathrm{CDCl}_{3}, 25^{\circ} \mathrm{C}, \mathrm{TMS}\right): \delta=10.42$ (brs, $1 \mathrm{H}$ ), 7.02 (s, $1 \mathrm{H}), 6.96(\mathrm{~s}, 1 \mathrm{H}), 6.94(\mathrm{~d}, 1 \mathrm{H}, J 1.6 \mathrm{~Hz}), 6.87$ (brd, $1 \mathrm{H}, J 8.6 \mathrm{~Hz}), 4.84(\mathrm{dd}, 1 \mathrm{H}, J 5.4,8.7 \mathrm{~Hz}), 4.30-4.21(\mathrm{~m}, 2 \mathrm{H})$, $3.95(\mathrm{~s}, 3 \mathrm{H}), 3.92(\mathrm{~s}, 3 \mathrm{H}), 2.34-2.25(\mathrm{~m}, 1 \mathrm{H}), 1.30(\mathrm{t}, 3 \mathrm{H}, J 7.1 \mathrm{~Hz}), 1.07(\mathrm{~d}, 3 \mathrm{H}, J 6.8 \mathrm{~Hz}), 1.04(\mathrm{~d}, 3 \mathrm{H}, J 6.8 \mathrm{~Hz}) ;{ }^{13} \mathrm{C}$ NMR $\left(100 \mathrm{MHz}_{2} \mathrm{CDCl}_{3}\right): \delta=172.4,161.9,149.3,145.9,131.9,128.6,120.3,103.2,102.1,94.4,61.6,57.3,56.0$, $55.8,31.5,19.2,18.1,14.3$; FT-IR (KBr) $\cup_{\max } 3376,3278,1733,1627,1539 \mathrm{~cm}^{-1}$. HRMS (ESI-TOF) $\mathrm{m} / \mathrm{z}:[\mathrm{M}+\mathrm{H}]^{+}$ calcd for $\mathrm{C}_{18} \mathrm{H}_{25} \mathrm{~N}_{2} \mathrm{O}_{5} 349.1763$; found: 349.1770 .

Ethyl (2S)-2-\{[(5-fluoro-1H-indol-2yl)carbonyl]amino\}-3-methylbutanoate, 3i: white solid; yield 47\%; m.p. $142-145^{\circ} \mathrm{C} ;[\alpha]_{\mathrm{D}}^{21}=+26.7$ (c 1.2, $\left.\mathrm{CHCl}_{3}\right) .{ }^{1} \mathrm{H} \mathrm{NMR}\left(400 \mathrm{MHz}, \mathrm{CDCl}_{3}, 25^{\circ} \mathrm{C}, \mathrm{TMS}\right): \delta=9.69$ (brs, $\left.1 \mathrm{H}\right), 7.40$ (dd, $1 \mathrm{H}, J$ 4.3, 8.9 Hz), $7.31(\mathrm{dd}, 1 \mathrm{H}, J$ 2.0, $9.1 \mathrm{~Hz}), 7.07(\mathrm{dt}, 1 \mathrm{H}, J$ 2.3, $9.1 \mathrm{~Hz}), 6.95(\mathrm{brs}, 1 \mathrm{H}), 6.77(\mathrm{brd}, 1 \mathrm{H}, J 8.6 \mathrm{~Hz}), 4.78$ (dd, $1 \mathrm{H}, J 5.0,8.7 \mathrm{~Hz}), 4,35-4,23(\mathrm{~m}, 2 \mathrm{H}), 2.38-2.28(\mathrm{~m}, 1 \mathrm{H}), 1.34(\mathrm{t}, 3 \mathrm{H}, J 7.1 \mathrm{~Hz}), 1.11-1.02(\mathrm{~m}, 6 \mathrm{H}) ;{ }^{13} \mathrm{C} \mathrm{NMR}$ $\left(100 \mathrm{MHz}, \mathrm{CDCl}_{3}\right): \delta=171.8,161.2,158.1\left(\mathrm{~d}, J_{\mathrm{C}-\mathrm{F}} 234.9 \mathrm{~Hz}\right), 133.0,131.7,127.7,113.6\left(\mathrm{~d}, J_{\mathrm{C}-\mathrm{F}} 26.6 \mathrm{~Hz}\right), 112.9$ (d, $\left.J_{C-F} 9.6 \mathrm{~Hz}\right), 106.2\left(\mathrm{~d}, J_{C-F} 23.3 \mathrm{~Hz}\right), 102.5\left(\mathrm{~d}, J_{\mathrm{C}-\mathrm{F}} 5.2 \mathrm{~Hz}\right), 61.5,57.3,31.7,18.9,17.9,14.2 ; \mathrm{FT}-\mathrm{IR}(\mathrm{KBr}) \mathrm{U}_{\max } 3355$, 3257, 1723, 1646, $1542 \mathrm{~cm}^{-1}$. HRMS (ESI-TOF) $\mathrm{m} / \mathrm{z}$ : $[\mathrm{M}+\mathrm{H}]^{+}$calcd for $\mathrm{C}_{16} \mathrm{H}_{20} \mathrm{FN}_{2} \mathrm{O}_{3}$ 307.1458; found: 307.1467.

Ethyl (2S)-3-methyl-2-\{[(5-nitro-1H-indol-2yl)carbonyl]amino\}butanoate, 3j: Yellow solid; yield 79\%; m.p. $119-122^{\circ} \mathrm{C} ;[\alpha]^{21}{ }_{\mathrm{D}}=-9.3$ (c 1.1, $\mathrm{CHCl}_{3}$ ). ${ }^{1} \mathrm{H}$ NMR $\left(400 \mathrm{MHz}, \mathrm{CDCl}_{3}, 25^{\circ} \mathrm{C}, \mathrm{TMS}\right): \delta=10.50(\mathrm{brs}, 1 \mathrm{H}), 8.29(\mathrm{~d}, 1 \mathrm{H}, \mathrm{J}$ $8.0 \mathrm{~Hz}$ ), $8.04(\mathrm{~d}, 1 \mathrm{H}, J 7.8 \mathrm{~Hz}), 7.30(\mathrm{t}, 1 \mathrm{H}, J 8.0 \mathrm{~Hz}), 7.12(\mathrm{~d}, 1 \mathrm{H}, J 2.3 \mathrm{~Hz}), 6.85(\mathrm{brd}, 1 \mathrm{H}, J 8.6 \mathrm{~Hz}), 4.82(\mathrm{dd}, 1 \mathrm{H}, J$ 4.8, 8.6 Hz), 4.33-4.25 (m, 2H), 2.37-2.31 (m, 1H), $1.36(\mathrm{t}, 3 \mathrm{H}, J 7.1 \mathrm{~Hz}), 1.06(\mathrm{~d}, 3 \mathrm{H}, J 6.9 \mathrm{~Hz}), 1.03(\mathrm{~d}, 3 \mathrm{H}, J 6.9$ $\mathrm{Hz}) ;{ }^{13} \mathrm{C} \mathrm{NMR}\left(100 \mathrm{MHz}, \mathrm{CDCl}_{3}\right): \delta=171.9,160.1,133.4,132.8,131.2,130.0,129.2,121.8,120.1,103.6,61.7$, 57.4, 31.8, 19.0, 17.9, 14.2; FT-IR (ATR) $U_{\max } 3467,3439,1749,1673,1548 \mathrm{~cm}^{-1}$. HRMS (ESI-TOF) $\mathrm{m} / \mathrm{z}:[\mathrm{M}+\mathrm{H}]^{+}$ calcd for $\mathrm{C}_{16} \mathrm{H}_{20} \mathrm{~N}_{3} \mathrm{O}_{5} 334.1403$; found: 334.1409 .

Ethyl (2S)-2-[(1H-indol-3-ylacetyl)amino]-3-methylbutanoate, 3k: brown oil; yield 44\%; $[\alpha]^{20}=+18.0$ (c 1.0, $\left.\mathrm{CHCl}_{3}\right) .{ }^{1} \mathrm{H}$ NMR $\left(400 \mathrm{MHz}, \mathrm{CDCl}_{3}, 25^{\circ} \mathrm{C}, \mathrm{TMS}\right): \delta=8.99$ (brs, $\left.1 \mathrm{H}\right), 7.60(\mathrm{~d}, 1 \mathrm{H}, J 7.8 \mathrm{~Hz}), 7.39(\mathrm{~d}, 1 \mathrm{H}, J 8.1 \mathrm{~Hz})$, $7.23(\mathrm{t}, 1 \mathrm{H}, J 7.9 \mathrm{~Hz}), 7.15(\mathrm{t}, 1 \mathrm{H}, J 8.0 \mathrm{~Hz}), 7.08(\mathrm{brs}, 1 \mathrm{H}), 6.30(\mathrm{~d}, 1 \mathrm{H}, J 8.8 \mathrm{~Hz}), 4.56(\mathrm{dd}, 1 \mathrm{H}, J 5.0,8.8 \mathrm{~Hz}), 4.17-$ $4.05(\mathrm{~m}, 2 \mathrm{H}), 3.80(\mathrm{~d}, 2 \mathrm{H}, J 2.4 \mathrm{~Hz}), 2.12-2.01(\mathrm{~m}, 1 \mathrm{H}), 1.21(\mathrm{t}, 3 \mathrm{H}, J 7.1 \mathrm{~Hz}), 0.84(\mathrm{~d}, 3 \mathrm{H}, J 6.8 \mathrm{~Hz}), 0.69(\mathrm{~d}, 3 \mathrm{H}, J$ $6.8 \mathrm{~Hz}) ;{ }^{13} \mathrm{C} \mathrm{NMR}\left(100 \mathrm{MHz}, \mathrm{CDCl}_{3}\right): \delta=171.9(2 \mathrm{C}), 136.3,126.9,124.2,122.3,119.9,118.5,111.5,108.1,61.4$, 57.2, 33.5, 31.0, 18.9, 17.5, 14.0; FT-IR (ATR) $U_{\max }$ 3405, 3303, 1731, 1651, 152

$1 \mathrm{~cm}^{-1}$. HRMS (ESI-TOF) $\mathrm{m} / \mathrm{z}$ : $[\mathrm{M}+\mathrm{H}]+$ calcd for $\mathrm{C}_{17} \mathrm{H}_{23} \mathrm{~N}_{2} \mathrm{O}_{3} 303.1709$; found: 303.1714 . 
Methyl (2S,3S)-2-[(1H-indol-3-ylacetyl)amino]-3-methylpentanoate, 31: colourless oil; yield $72 \% ;[\alpha]^{21}=$ $+11.2\left(\mathrm{c} 0.9, \mathrm{CHCl}_{3}\right) .{ }^{1} \mathrm{H} \mathrm{NMR}\left(400 \mathrm{MHz} \mathrm{CDCl}_{3}, 25^{\circ} \mathrm{C}, \mathrm{TMS}\right): \delta=8.87$ (brs, $\left.1 \mathrm{H}\right), 7.60(\mathrm{~d}, 1 \mathrm{H}, J 7.8 \mathrm{~Hz}), 7.37(\mathrm{~d}, 1 \mathrm{H}$, J $8.0 \mathrm{~Hz}$ ), 7.24-7.10 (m, 2H), $7.08(\mathrm{~s}, 1 \mathrm{H}), 6.28(\mathrm{brd}, 1 \mathrm{H}, J 8.5 \mathrm{~Hz}), 4.60(\mathrm{dd}, 1 \mathrm{H}, J 5.2,8.6 \mathrm{~Hz}), 3.78(\mathrm{~s}, 2 \mathrm{H}), 3.66$ $(\mathrm{s}, 3 \mathrm{H}), 1.80-1.76(\mathrm{~m}, 1 \mathrm{H}), 1.28-1.22(\mathrm{~m}, 1 \mathrm{H}), 1.0-0.89(\mathrm{~m}, 1 \mathrm{H}), 0.88-0.75(\mathrm{~m}, 6 \mathrm{H}) ;{ }^{13} \mathrm{C} \mathrm{NMR}\left(100 \mathrm{MHz}^{\mathrm{C}} \mathrm{CDCl}\right): \delta$ = 172.6, 170.9, 136.3, 126.9, 123.9, 122.6, 119.9, 118.9, 111.5, 109.2, 56.5, 51.8, 37.3, 33.3, 24.6, 15.6, 11.5; FT-IR (ATR) $U_{\max } 3467,3355,1744,1651,1518 \mathrm{~cm}^{-1}$. HRMS (ESI-TOF) $\mathrm{m} / \mathrm{z}$ : $[\mathrm{M}+\mathrm{H}]^{+}$calcd for $\mathrm{C}_{17} \mathrm{H}_{23} \mathrm{~N}_{2} \mathrm{O}_{3}$ 303.1709; found: 303.1716.

Ethyl (2S)-3-methyl-2-[(1H-pirrol-2-ylcarbonyl)amino]butanoate, 3m: ${ }^{44}$ white solid; yield 66\%; m.p. 109$114^{\circ} \mathrm{C} ;{ }^{1} \mathrm{H} \mathrm{NMR}\left(400 \mathrm{MHz}, \mathrm{CDCl}_{3}, 25^{\circ} \mathrm{C}, \mathrm{TMS}\right): \delta=9.80$ (brs, $\left.1 \mathrm{H}\right), 6.96-6.91(\mathrm{~m}, 1 \mathrm{H}), 6.72-6.66(\mathrm{~m}, 1 \mathrm{H}), 6.45(\mathrm{~d}$, $1 \mathrm{H}, J 8.4 \mathrm{~Hz}), 6.28-6.22(\mathrm{~m}, 1 \mathrm{H}), 4.72(\mathrm{dd}, 1 \mathrm{H}, J 5.0,8.8 \mathrm{~Hz}), 4.30-4.18(\mathrm{~m}, 2 \mathrm{H}), 2.31-2.19(\mathrm{~m}, 1 \mathrm{H}), 1.30(\mathrm{t}, 3 \mathrm{H}, J$ $7.1 \mathrm{~Hz}), 1.00(\mathrm{~d}, 3 \mathrm{H}, J 6.9 \mathrm{~Hz}), 0.98(\mathrm{~d}, 3 \mathrm{H}, J 6.9 \mathrm{~Hz}) ;{ }^{13} \mathrm{C} \mathrm{NMR}\left(100 \mathrm{MHz}, \mathrm{CDCl}_{3}\right): \delta=170.5,159.2,123.8,120.2$, $108.1,107.8,59.7,55.2,30.0,17.3,16.2,12.5$.

Methyl (2S)-3-phenyl-2-[(1H-pirrol-2-ylcarbonyl)amino]propanoate, 3n: ${ }^{45}$ white solid; yield 58\%; m.p. 97$100^{\circ} \mathrm{C} ;[\alpha]_{D}^{21}=+27.2$ (c 1.0, $\mathrm{CHCl}_{3}$ ). ${ }^{1} \mathrm{H}$ NMR (400 MHz, CDCl $\left.3,25^{\circ} \mathrm{C}, \mathrm{TMS}\right): \delta=10.91$ (brs, $\left.1 \mathrm{H}\right), 7.39-7.27(\mathrm{~m}$, $5 \mathrm{H}), 7.21(\mathrm{~d}, 1 \mathrm{H}, \mathrm{J} 7.9 \mathrm{~Hz}), 6.96(\mathrm{~s}, 1 \mathrm{H}), 6.75(\mathrm{~s}, 1 \mathrm{H}), 6.24(\mathrm{~s}, 1 \mathrm{H}), 5.24-5.17(\mathrm{~m}, 1 \mathrm{H}), 3.66(\mathrm{~s}, 3 \mathrm{H}), 3.38-3.25(\mathrm{~m}$, $2 \mathrm{H}) ;{ }^{13} \mathrm{C} \mathrm{NMR}\left(100 \mathrm{MHz}, \mathrm{CDCl}_{3}\right): \delta=172.8,161.4,136.4,129.3(2 \mathrm{C}), 128.9(2 \mathrm{C}), 127.1,125.9,125.2,122.6$, 110.6, 109.6, 53.5, 52.4, 38.0.

Antimicrobial activity Ten yeast strains belonging to six different species were employed in this study: Candida albicans CBS 562 (CMC 298), Candida albicans CMC 1968, Candida glabrata CBS 138 (CMC 2055), Candida glabrata CMC 1933, Candida parapsilosis CBS 604 (CMC 197), Candida parapsilosis CMC 2039, Candida tropicalis CBS 94 (CMC 2054), Candida tropicalis CMC 2041, Meyerozyma guilliermondii CBS 2030 (CMC 1500) and Saccharomyces cerevisiae CMC 520. Isolates are kept frozen at $-80^{\circ} \mathrm{C}$ in $17 \%$ glycerol solution in the laboratory collection of the microbiology section of the Department of Pharmaceutical Sciences, University of Perugia (Italy). Short term storage was carried out at $4^{\circ} \mathrm{C}$ on YEPDA (YEPD added with $1.7 \%$ agarose). Yeast strains were grown in YEPD [Yeast Extract 1\%, Peptone 1\%, Dextrose 1\% - all products from Biolife (Italy)] at $37^{\circ} \mathrm{C}$ with $150 \mathrm{rpm}$ shaking. For each compound the following concentrations were tested: 0 $\mu \mathrm{g} / \mathrm{mL}, 15.6 \mu \mathrm{g} / \mathrm{mL}, 31.2 \mu \mathrm{g} / \mathrm{mL}, 62.5 \mu \mathrm{g} / \mathrm{mL}, 125 \mu \mathrm{g} / \mathrm{mL}, 250 \mu \mathrm{g} / \mathrm{mL}, 500 \mu \mathrm{g} / \mathrm{mL}$. Each compound was prepared as a 100X stock solution in DMSO and then serially diluted in RPMI medium to obtain the final working solution. The adopted experimental protocol was developed according to the EUCAST guidelines ${ }^{46}$ and briefly outlined in the following lines. Each strain was grown over night in bottles containing YEPD medium, at $37^{\circ} \mathrm{C}$ in an orbital shaker at $150-180 \mathrm{rpm}$ and then harvested and centrifuged at 3,000 $\times \mathrm{g}$ for 5 minutes at $4{ }^{\circ} \mathrm{C}$. The supernatant was removed, and the pellet was washed twice with sterile distilled water. Washed yeasts cells were then resuspended in RPMI-1640 with $2 \%$ glucose (Sigma Aldrich) medium, in order to obtain a final density of $1.0 \times 10^{6}$ cells $/ \mathrm{mL}$. $100 \mu \mathrm{L}$ of these standardized cell suspensions were seeded in each selected well of 96-well microtiter plates in which each studied compound was already aliquoted. A growth control well, consisting of microbial cells without any compound, and a negative control well, consisting of sterile RPMI medium, were also prepared. The microtiter plate was closed, sealed and incubated for $24 \mathrm{~h}$ at $37^{\circ} \mathrm{C}$. The plate was then recovered and using a multichannel pipette, $20 \mu \mathrm{l}$ of fresh $0.01 \% \mathrm{w} / \mathrm{v}$ resazurin aqueous solution were added to each well of the plate, included the control wells, to assess MIC. After $1 \mathrm{~h}$ incubation at $37^{\circ} \mathrm{C}$, the plate was visually inspected to highlight the presence of pink color gradient, resulting from the reduction of the blue dye resazurin to the pink dye resorufin by living cells. MIC value was assessed as the compound concentration present in the last well before a visible color change was detectable. 


\section{Acknowledgements}

University of Perugia "Fondo Ricerca di base 2018", MIUR "Finanziamento annuale individuale delle attività base di ricerca FFABR 2017". This manuscript is part of the scientific activity of the international network Selenium Sulfur Redox and catalysis and national Interuniversity Consortium C.I.N.M.P.I.S.

\section{Supplementary Material}

NMR spectra are available as supplementary material of this article.

\section{References}

1. Greenberg, A.; Breneman, C.M.; Liebman, J.F. The amide Linkage: Selected Structural Aspects in Chemistry, Biochemistry and Materials Science (Wiley-Interscience, 2000).

2. Pattabiraman, V.R.; Bode, J.W. Nature 2011, 480, 471-479.

https://doi.10.1038/nature10702

3. Scheidt, K. Nature 2010, 465, 1020-1022.

https://doi.org/10.1038/4651020a

4. Gernigon, N.; Al-Zoubi, R.M.; Hall, D.G. J. Org. Chem. 2012, 77, 8386-8400.

https://dx.doi.org/10.1021/jo30132581

5. Ghose., A K.; Viswanadhan, V.N.; Wendoloski, J.J. J. Comb. Chem. 1999, 1, 55-68.

https://www.ncbi.nlm.nih.gov/pubmed/10746014

6. Roughley, S.D.; Jordan, A.M. J. Med. Chem. 2011, 54, 3451-3479.

https://dx.doi.org/10.1021/im200187y

7. Srivastava, V.; Singh, P.K.; Singh, P.P. Tetrahedron Lett. 2019, 60, 40-43 and references cited therein. https://dx.doi.org/10.1016/jtetlet.2018.11.050

8. Constable, D.J.C.; Dunn, P. J.; Hayler, J.D.; Humphrey, G.R.; Leazer, J.L. Jr; Linderman, R.J.; Lorenz, K.; Manley, J; Pearlman B.A.; Wells, A.; Zaks, A.; Zhang, T.Y. Green Chem. 2007, 9, 411-420.

https://doi/10.1039/b703488c

9. Dunn, P.J.; Galvin, S.; Hettembach, K. Green Chem. 2004, 6, 43-48.

https://doi/10.1039/b312329d

10. Bandini, M.; Eichholzer, A. Angew. Chem. Int. Ed. 2009, 48, 9608-9644.

https://doi/10.1002/anie.200901843

11. Sweidan, K.; Sabbah, D.A.; Bardaweel, S.; Dush, K.A.; Sheikha, G.A.; Mubarak, M.S. Bioorg. Med. Chem. Lett. 2016, 26, 2685-2690.

https://dx.doi.org/10.1016/i.bmcl.2016.04.011

12. Milkiewicz, K.L.; Marsilje, T.H.; Woodworth, R P. Jr.; Bifulco, N. Jr.; Hangauer, M.J.; Hangauer, D.G. Bioorg. Med. Chem. Lett. 2000, 10, 483-486.

https://doi.org/10.1016/S0960-894X(00)00039-1

13. Youssif, B.G M.; Abdelrahman, M.H.; Abdelazeem, A.H.; Abdelgawad, M.A.; Ibrahim, H.M.;. Salem, O.I.A.; Mohamed, M.F.A.; Treambleau, L.; Bukhari, S.N.A. Eur. J. Med. Chem. 2018, 146, 260-273.

https://doi.org/10.1016/j.ejmech.2018.01.042 
14. Cruz-Lopez, O.; Diaz-Mochon, J. J.; Campos, J.M.; Entrena, A.; Nunez, M.T.; Labeaga, L.; Orjales, A.; Gallo, M.A.; Espinosa, A. ChemMedChem 2007, 2, 88-100.

dhttps://doi:10.1002/cmdc.200600179

15. Liu, Z.; Tang. L.; Zhu, H.; Xu, T.; Qiu, C.; Zheng, S.; Gu, Y.; Feng, J.; Zhang, Y.; Liang, G. J. Med. Chem. 2016, 59, 4637-4650.

https://doi:10.1021/acs.jmedchem.5b02006

16. Penning, T.D.; Talley, J.J.; Bertenshaw, S.R.; Carter, J.S.; Collins, P.W.; Docter, S.; Graneto, M.J.; Lee, L.F.; Malecha, J.W.; Miyashiro, J.M.; Rogers, R.S.; Rogier, D.J.; Yu, S.S.; Anderson, G.D.; Burton, E.G.; Cogburn, J.N.; Gregory, S.A.; Koboldt, C M.; Perkins, W.E.; Seibert, K.; Veenhuizen, A.W.; Zhang, Y.Y.; Isakson, P.C. J. Med. Chem. 1997, 40, 1347-1365.

https://doi.org/10.1021/jm960803q

17. Kondreddi, R.R.; Jiricek, J.; Rao, S.P.S.; Lakshminarayana, S.B.; Camacho, L.R.; Rao, R.; Herve, M.; Bifani, P.; Ma, N. L.; Kuhen, K.; Goh, A.; Chatterjee, A.K.; Dick, T., Diagana, T.T., Manjunatha, U.H.; Smith, P.W. Chatterjee, A.K., Dick, T. ; Diagana, T.T. ; Manjunatha, U.H. ; Smith P.W. J. Med. Chem. 2013, 56, 88498859.

https://dx.doi.org/10.1021/jm4012774I

18. Stec, J; Onajole, O.K.; Lun, S.; Guo, H., Merenbloon, B.; Vistoli, G., Bishai, W.R.; Kozikowski, A.P. J. Med. Chem. 2016, 59, 6232-6247.

https://doi.org/10.1021/acs.jmedchem.6b00415

19. Patel, H. M. Green and Sustainable Chemistry 2015, 5, 137-144.

http://dx.doi.org/10.4236/gsc.2015.54017

20. Mane, Y.D.; Patil, S.S.; Biradar, D.O.; Khade, B.C. Heterocycl. Commun. 2018, 24, 327-332. https://doi.org/10.1515/hc-2018-0107

21. Patel, H. M. Curr. Bioact. Compd. 2018, 14, 278-288. https://doi.org/10.2174/1573407213666170424164716

22. Patel, H.M.; Rajani, D.P.; Sharma, M.G.; Bhatt. H.G. Lett. Drug Des. Discov. 2019, 16, 119 - 126. https://doi.org/10.2174/1570180815666180502123743

23. Zhao, S.; Wei, P.; Wu, M.; Zhang, X.; Zhao, L.; Jiang, X.; Hao, C.; Su, X.; Zhao, D.; Cheng, M. Bioorg. Med. Chem. 2018, 26, 3242-3253.

https://doi/10.1515/znc-2008-3-405

24. Olgen, S.; Altanlar, N., Karatayli, E., Bozdayi, M. Z.Naturforsch C. 2008, 63, 189-195. https://doi/10.1515/znc-2008-3-405

25. Pandolfi, F.; D’Acierno, F., Bortolami, M.; De Vita, D.; Gallo, F.; De Meo, A.; Di Santo, R.; Costi, R.; Simonetti, G.; Scipione, L. Eur. J. Med. Chem. 2019, 165, 93-106. https://doi.org/10.1016/j.ejmech.2019.01.012

26. Patel, H.M.; Patel, K.D.; Patel. H D. Curr. Bioact. Compd. 2017, 13, 47-58. https://doi.org/10.2174/1573407212666160517145130

27. Famiglini, V.; La Regina, G.; Coluccia, A.; Pelliccia, S.; Brancale, A.; Maga, G.; Crespan, E.; Badia, R.; RiveiraMunoz, E., Esté, J.A.; Ferretti, R.; Cirilli, R.; Zamperini, C.; Botta, M.; Schols, D.; Limongelli, V.; Agostino, B.; Novellino, E.; Silvestri, R. J. Med. Chem. 2014, 57, 9945-9957.

https://dx.doi.org/10.1021/im5011622I

28. Ban, F.; Leblanc, E.; Li, H.; Munuganti, R.S.N.; Frewin, K.; Rennie, P.S.; Cherkasov, A. J. Med. Chem. 2014, 57, 6867-6872. 
29. Chen, K.X.; Vibulbhan, B.; Yang, W.; Sannigrahi, M.; Velazquez, F., Chan, T-Y.; Venkatraman, S.; Anilkumar, G.N.; Zeng, Q.; Bennet, F., Jiang, Y.; Lesburg, C.A.; Duca, J.; Pinto, P.; Gavalas, S., Huang, Y., Wu, W.; Selyutin, O.; Agrawal, S.; Feld, B., Huang, H.-C.; Li, C., Cheng, K.-C., Shih, N.-Y.; Kozlowski, J. A.; Rosenblum, S. B., Njoroge, F.G. J. Med. Chem. 2012, 55, 754-765.

https://dx.doi.org/10.1021/jm201258kl

30. Liu, G., Zhang, Z., Luo, X.; Shen, J.; Liu, H.; Shen, X.; Chen, K., Jiang, H. Bioorg. Med. Chem. 2004, 12, 41474157.

https://doi:10.1016/j.bmc.2004.05.023

31. La Regina, G.; Silvestri, R.; Gatti, V.; Lavecchia, A.; Novellino, E.; Befani, O.; Turini, P.; Agostinelli, E. Bioorg. Med. Chem. 2008, 16, 9729-9740.

https://doi:10.1016/j.bmc.2008.09.072

32. La Regina, G.; Silvestri, R.; Artico, M.; Lavecchia, A.; Novellino, E.; Befani, O.; Turini, P.; Agostinelli, E. J. Med. Chem. 2007, 50, 922-931.

https://doi:10.1021/jm060882y

33. Silvestri, R.; La Regina, G.; De Martino, G.; Artico, M.; Befani, O.; Palumbo, M., Agostinelli, E.; Turini, P. J. Med. Chem. 2003, 46, 917-920.

htpps://doi:10.1021/jm0256124

34. Maolanon, A.R.; Risgaard, R.; Wang, S.-Y.; Snoep, Y.; Papangelis, A.; Yi, F.; Holley, D.; Barslund, A.F.; Svenstrup, N.; Hansen, K.B.; Clausen, R.P. ACS Chem. Neurosci. 2017, 8, 1681-1687.

https://dx.doi.org/10.1021/acschemneuro.7b00117

35. Thanigaimalai, P.; Konno, S.; Yamamoto, T.; Koiwai, Y.; Taguchi, A.; Takayana, K.; Yakushiji, F.; Akaji, K.; Chen, S.-E.; Naser-Tavakolian, A.; Schon, A.; Freire, E.; Hayashi, Y. Eur. J. Med. Chem. 2013, 68, 372-384. https://doi/10.1016/i.ejmech.2013.07.037

36. de Figueiredo R.M.; Suppo, J.-S.; Campagne, J.-M. Chem. Rev. 2016, 116, 12029-12122. https://doi/10.1021/acs.chemrev.6b00237

37. Valeur, E.; Bradley, M. Chem. Soc. Rev. 2009, 38, 606-631. https://doi/10.1039/b701677h

38. Montalbetti, C.A.G.N.; Falque, V.M. Wiley Encyclopedia of chemical biology 2008, 2171-2187. https://doi:1002/9780470048672.wecb635

39. Montalbetti, C.A.G.N.; Falque, V.M. Tetrahedron 2005, 61, 10827-10852.

https://doi/1016/j.tet.2005.08.031

40. Allen, C.L.; Williams, J.M.J. J. Chem. Soc. Rev. 2011, 40, 3405-3415. https://doi:10.1039/c0cs00196a

41. Lundberg, H.; Tinnis, F.; Selander, N.; Adolfsson, H. Chem. Soc. Rev. 2014, 43, 2714-2742. https://doi/10.1039/c3cs60345h

42. Joullié, M. M.; Lassen, K. M. Arkivoc 2010, 189-250.

http://dx.doi.org/10.3998/ark.5550190.0011.816

43. König, W.; Geiger, R. Chem. Ber. 1970, 103, 788-798. http://dx.doi.org/10.1002/cber.19701030319

44. Palomba, M.; Sancineto, L.; Marini, F.; Santi, C.; Bagnoli, L. Tetrahedron 2018, 74,7156-7163. https://doi/10.1016/i.tet.2018.10.044

45. Tian, H.; Ermolenko, L.; Gabant, M.; Vergne, C.; Moriou, C.; Retailleau, P.; Al-Mourabit, A. Adv. Synth Catal. 2011, 353, 1525-1533. 
46. Arendrup, M.C.; Cuenca-Estrella, M.; Lass-Florl, C.; Hope, W. and the EUCAST-AFST Clin. Microbiol. Infect. 2012, 18 E246-247. https://doi/10.111/j.1469-0691.2012.03880.x

This paper is an open access article distributed under the terms of the Creative Commons Attribution (CC BY) license (http://creativecommons.org/licenses/by/4.0/) 